\title{
Thermal Resistance across Interfaces Comprising Dimensionally Mismatched Carbon Nanotube-Graphene Junctions in 3D Carbon Nanomaterials
}

\author{
Jungkyu Park and Vikas Prakash \\ Department of Mechanical and Aerospace Engineering, Case Western Reserve University, Cleveland, OH 44106-7222, USA \\ Correspondence should be addressed to Vikas Prakash; vikas.prakash@case.edu
}

Received 17 December 2013; Accepted 1 April 2014; Published 25 June 2014

Academic Editor: Naoki Kishi

Copyright (C) 2014 J. Park and V. Prakash. This is an open access article distributed under the Creative Commons Attribution License, which permits unrestricted use, distribution, and reproduction in any medium, provided the original work is properly cited.

In the present study, reverse nonequilibrium molecular dynamics is employed to study thermal resistance across interfaces comprising dimensionally mismatched junctions of single layer graphene floors with $(6,6)$ single-walled carbon nanotube (SWCNT) pillars in 3D carbon nanomaterials. Results obtained from unit cell analysis indicate the presence of notable interfacial thermal resistance in the out-of-plane direction (along the longitudinal axis of the SWCNTs) but negligible resistance in the inplane direction along the graphene floor. The interfacial thermal resistance in the out-of-plane direction is understood to be due to the change in dimensionality as well as phonon spectra mismatch as the phonons propagate from SWCNTs to the graphene sheet and then back again to the SWCNTs. The thermal conductivity of the unit cells was observed to increase nearly linearly with an increase in cell size, that is, pillar height as well as interpillar distance, and approaches a plateau as the pillar height and the interpillar distance approach the critical lengths for ballistic thermal transport in SWCNT and single layer graphene. The results indicate that the thermal transport characteristics of these SWCNT-graphene hybrid structures can be tuned by controlling the SWCNT-graphene junction characteristics as well as the unit cell dimensions.

\section{Introduction}

Based on the exceptional thermal transport characteristics as well as the electronic mobility of one- and two-dimensional carbon nanomaterials (e.g., carbon nanotube and graphene), there have been several attempts by researchers to synthesize three-dimensional (3D) hybrid nanostructures that synergistically combine two or more low-dimensional carbon nanomaterials [1-7]. Amongst the several potential 3D carbon nanostructured materials, 3D pillared single-walled carbon nanotube- (SWCNT-) graphene architectures (PGS) that incorporate SWCNTs as pillars and single layer graphene as floors have attracted particular attention $[1,4,6,8,9]$. These materials have the potential to exploit the extraordinary thermal conductivity of their base nanomaterials to enable tunable thermal transport in both out-of-plane and in-plane directions. Varshney et al. [1], using molecular dynamics, were the first to report thermal transport properties of PGS. More recently, Loh et al. [10] have shown that long wavelength out-of-plane phonon modes contribute significantly to thermal transport in such structures.

In the present paper we focus attention on thermal resistance across dimensionally mismatched SWCNT-graphene junctions in the 3D PGS. Using classical molecular dynamics with adaptive intermolecular reactive empirical bond order (AIREBO) interatomic potential interfacial thermal resistances are computed and the thermal conductivities of 3D PGS are compared with those of pristine SWCNT and single layer graphene of comparable size. The presence of the interfacial thermal resistance at the junctions is hypothesized to be due to the change in dimensionality (from nearly $1 \mathrm{D}$ to $2 \mathrm{D}$ to $1 \mathrm{D}$ ) as well as phonon spectra along the thermal transport path as the phonons propagate from the SWCNT to the graphene sheet and then back again to the SWCNT. In order to investigate this we compute the local density of states at various locations along the phonon transport path from SWCNT to graphene to SWCNT. 


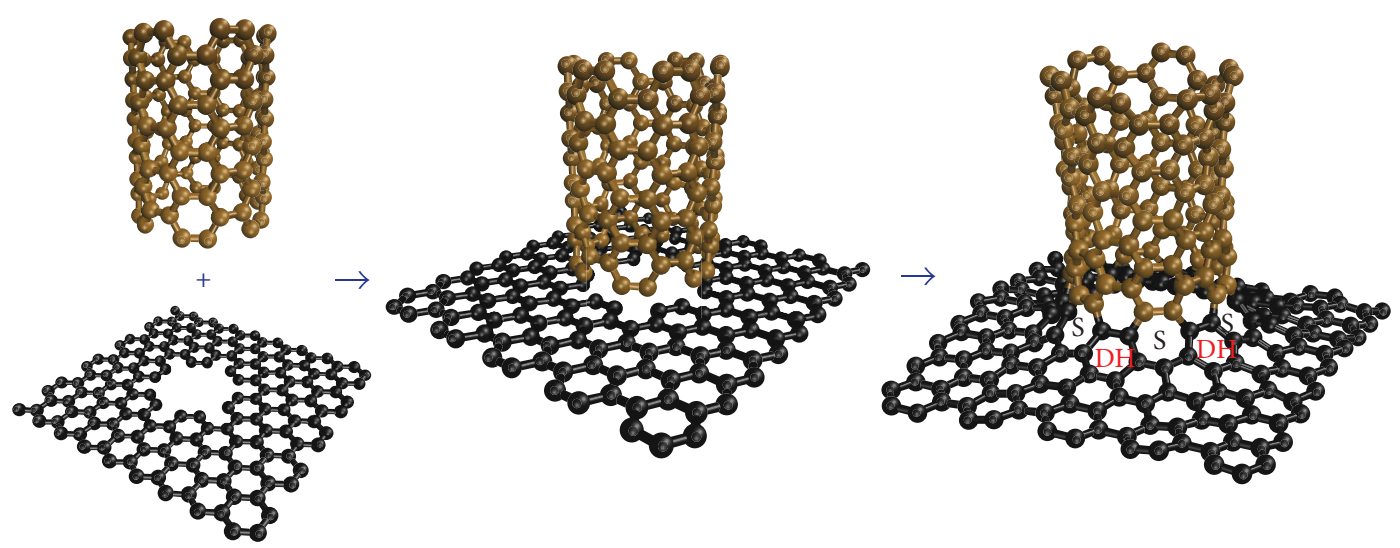

(a)

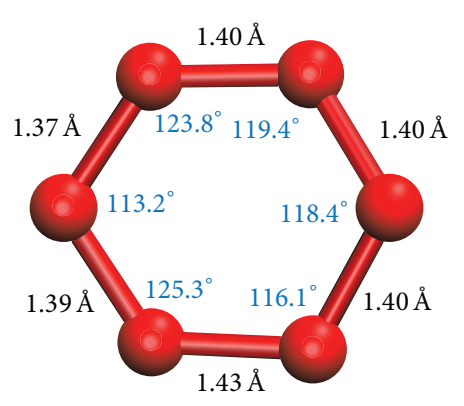

(b)

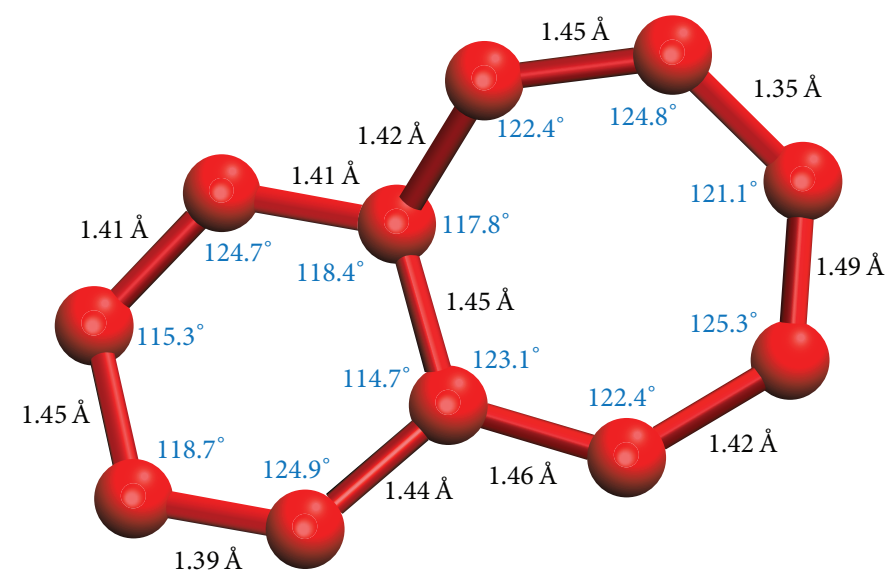

(c)

FIGURE 1: (a) Formation of a SWCNT-graphene junction. "S" denotes septagon and "DH" denotes distorted hexagon. (b) A hexagonal carbon ring in $(6,6)$ SWCNT. (c) A hexagon/septagon carbon ring in a SWCNT-graphene junction.

\section{Simulation Method}

In the present study, SWCNT-graphene junction structures were constructed by translating carbon atoms of $(6,6)$ armchair SWCNTs to match with corresponding holes in free standing single layer graphene nanostructures that were built using the visual molecular dynamics (VMD) [11] software. The procedure resulted in the creation of SWCNT-graphene junctions with regularly distributed septagon/hexagon carbon rings $[12,13]$ with $\mathrm{sp}^{2}$ type hybridization at the SWCNTgraphene junctions. Figure 1(a) illustrates the creation of such junction. It is well known that, in graphene, the three outershell electrons of each carbon atom occupy the planar $\mathrm{sp}^{2}$ hybrid orbital to form three in-plane $\sigma$ bonds with an out-ofplane $\pi$ orbital (bond). This makes graphene a planar hexagonal network. The length and energy of the $\sigma$ bonds in the $\mathrm{sp}^{2}$ hybridization are $0.14 \mathrm{~nm}$ and $420 \mathrm{kcal} / \mathrm{mol}$, respectively, and $0.15 \mathrm{~nm}$ and $360 \mathrm{kcal} / \mathrm{mol}$ in the $\mathrm{sp}^{3}$ configuration. The planar hexagonal structure along with the strong covalent $\left(\mathrm{sp}^{2}\right)$ bonding is understood to be responsible for the high in-plane phonon group velocities and thus high in-plane thermal conductivity of graphene [14]. When a graphene sheet is rolled to form a nanotube, the circular curvature of the nanotubes promotes quantum confinement and $\sigma-\pi$ rehybridization (i.e., $\sigma-\pi$ bond mixing); the three planar $\sigma$ bonds are forced slightly out of plane and for compensation the $\pi$ orbital is more delocalized outside the tube. This rehybridization together with the $\pi$ electrons confinement gives nanotubes their unique, extraordinary mechanical, thermal, and electronic properties. Moreover, they allow for topological defects, such as pentagons and septagons, to be incorporated into the hexagonal networks to form bent, toroidal, and helical nanotubes. For the purposes of the present study, the formation of the SWCNT-graphene junctions with $\mathrm{sp}^{2}$ hybridized covalent bonds between SWCNT and graphene involves creating six septagonal and six distorted hexagonal carbon rings, as shown in Figure 1(a); the existence of these seven member rings and distorted hexagon rings in a SWCNT-graphene junction has been confirmed by experiments [9].

Figures 1(b) and 1(c) illustrate the detailed configuration of a hexagonal carbon ring in a $(6,6)$ SWCNT and a septagon/hexagon carbon ring in a SWCNT-graphene junction obtained from the molecular dynamics simulations. The average bond length in a hexagonal carbon ring of a $(6,6)$ SWCNT is $0.1398 \mathrm{~nm}$ while the average bond length in a 


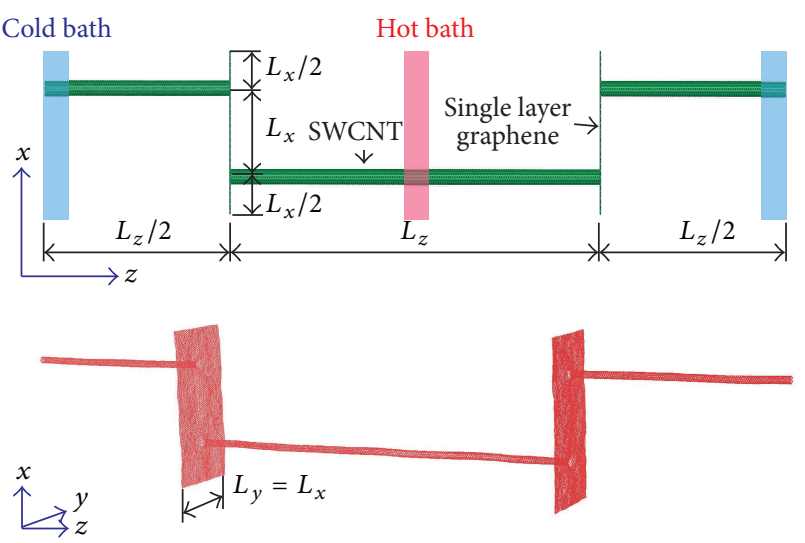

(a)

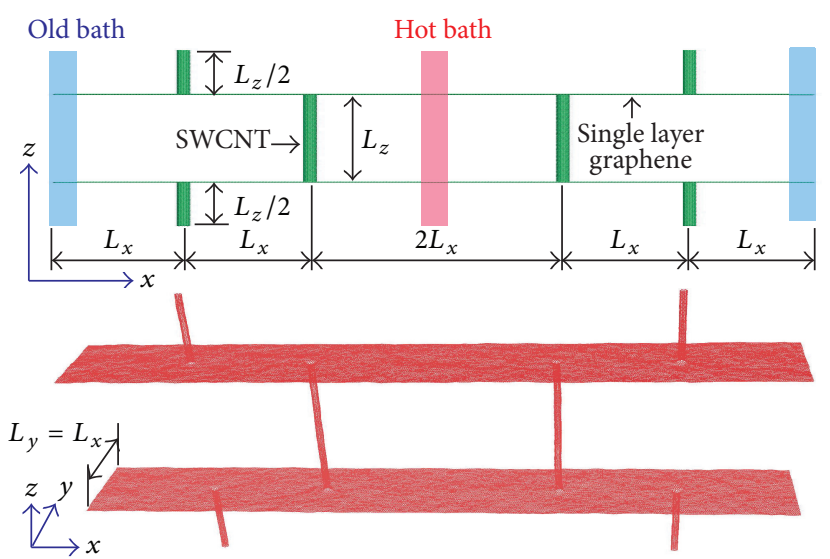

(b)

FiguRE 2: Schematic of sample preparation for RNEMD for (a) out-of-plane and (b) in-plane thermal conductivity calculations. $L_{x}$ denotes interpillar distance (length of graphene between two SWCNT pillars) and $L_{z}$ denotes pillar height (length of SWCNT between two graphene layers).

typical distorted hexagonal carbon ring from the SWCNTgraphene junction region is $0.1425 \mathrm{~nm}$. Moreover, the average bond length in a typical septagonal carbon ring from the SWCNT-graphene junction region is found to be $0.1434 \mathrm{~nm}$. Charlier et al. [15] using tight-binding models to investigate topological defects in SWCNTs estimated the bond length in a hexagon ring of a single 5/6/7 defect to be $0.1421 \mathrm{~nm}$ and the bond length in a septagon ring of a single 5/7 defect to be $0.1433 \mathrm{~nm}$. This confirms that the distorted hexagonal and septagonal carbon rings in SWCNT-graphene junctions have similar molecular structures to the topological defects observed in pristine SWCNTs and are expected to play the role of defects in SWCNTs $[16,17]$ giving rise to phonon scattering and thus an interfacial thermal resistance at the SWCNT-graphene junctions.

In the present research reverse nonequilibrium molecular dynamics (RNEMD) $[16,18-20]$ is used to estimate the thermal conductivities in the hybrid structures as well as the interfacial thermal resistance at the SWCNT-graphene junctions. Two types of simulation structures (unit cells), depicted in Figure 2, are constructed according to the direction in which thermal conductivity is to be evaluated. Temperature profiles are obtained along the SWCNT axis, that is, along the $z$ direction, as shown in Figure 2(a), to investigate the effect of SWCNT-graphene junction on the thermal transport characteristics in the cross-plane direction. We will denote this as "out-of-plane" thermal conductivity. On the other hand, the "in-plane" thermal conductivity is defined as the thermal conductivity along the graphene layer, that is, along the $x$ direction in Figure 2(b), which is useful for understanding the effects of SWCNT-graphene junction on thermal transport (phonon scattering) along the graphene layers. Two control parameters used in the construction of SWCNTgraphene junction structures are the pillar height, $L_{z}$, and the interpillar distance, $L_{x}$. Pillar height is the length of the SWCNT including the junction region that connects the two adjacent graphene floors. It also determines the sample length for thermal conductivity calculations in the out-of-plane direction (Figure 2(a)). Three different pillar heights, that is, $L_{z}=50 \mathrm{~nm}, 100 \mathrm{~nm}$, and $200 \mathrm{~nm}$, are chosen for the study. Interpillar distance, $L_{x}$, is defined as the distance between two SWCNT-graphene junctions and determines the sample length for the in-plane thermal conductivity calculations-the sample size is three times the interpillar distance (Figure 2(b)). For the four interpillar distances $\left(L_{x}\right.$ $=5 \mathrm{~nm}, 10 \mathrm{~nm}, 15 \mathrm{~nm}$, and $20 \mathrm{~nm}$ ) chosen in the present study, the in-plane thermal conductivity is calculated for four different sample sizes, that is, four different lengths of graphene layers, which are $15 \mathrm{~nm}, 30 \mathrm{~nm}, 45 \mathrm{~nm}$, and $60 \mathrm{~nm}$.

All simulations are performed using the large-scale atomic/molecular massively parallel simulator (LAMMPS) [21] code using the AIREBO [22] interatomic potential. The simulation structure is energy-minimized to reach the equilibrium configuration by iteratively adjusting atomic coordinates. After elevating the temperature $(500 \mathrm{~K})$ using isenthalpic (NPH) ensemble with Langevin thermostat for faster equilibration, the system is cooled to room temperature with the isothermal-isobaric (NPT) ensemble. RNEMD is carried out on the equilibrated structures for $2 \mathrm{~ns}$ with the microcanonical (NVE) ensemble with a time step of $1 \mathrm{fs}$. Figure 3 shows the total (cumulative) energy transported and the evolution of the temperature profile as a function of time for the SWCNT-graphene junction structure with $50 \mathrm{~nm}$ pillar height and $5 \mathrm{~nm}$ interpillar distance. As seen from the figure the system quickly approaches a steady statethe quick stabilization of heat flow and the temperature distribution is due to the well-equilibrated molecular structure. In addition, during RNEMD with NVE ensemble the total energy is found to be conserved adequately with only small energy fluctuations of $\sim 10 \mathrm{eV}$. These observations are true for all SWCNT-graphene junction structures utilized in this work. Also, in all simulated structures thermal conductivities and interfacial thermal resistances are obtained from the temperature profiles after $2 \mathrm{~ns}$ of RNEMD simulations to ensure achievement of steady state transport conditions. 


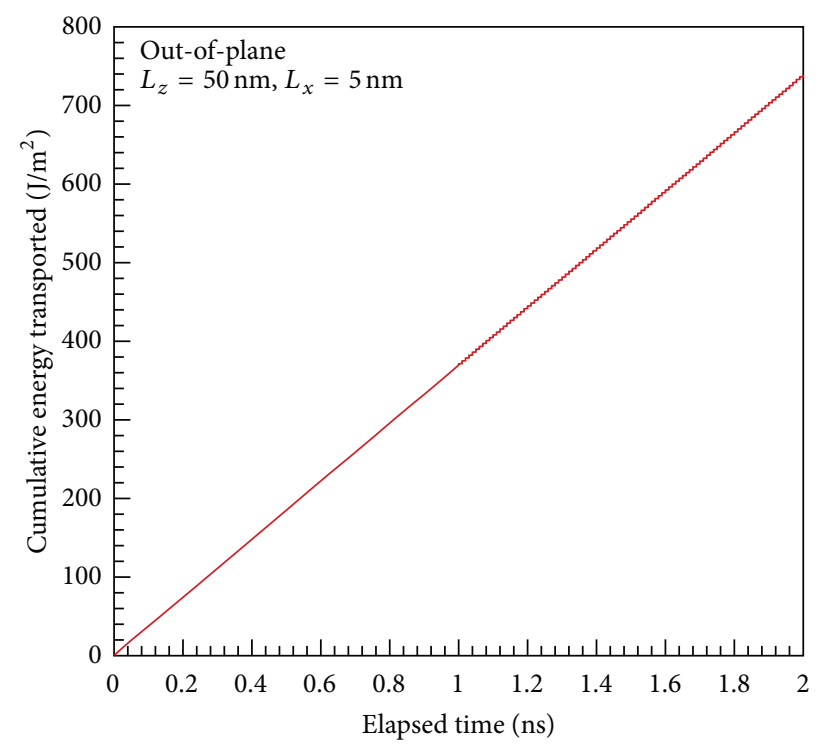

(a)

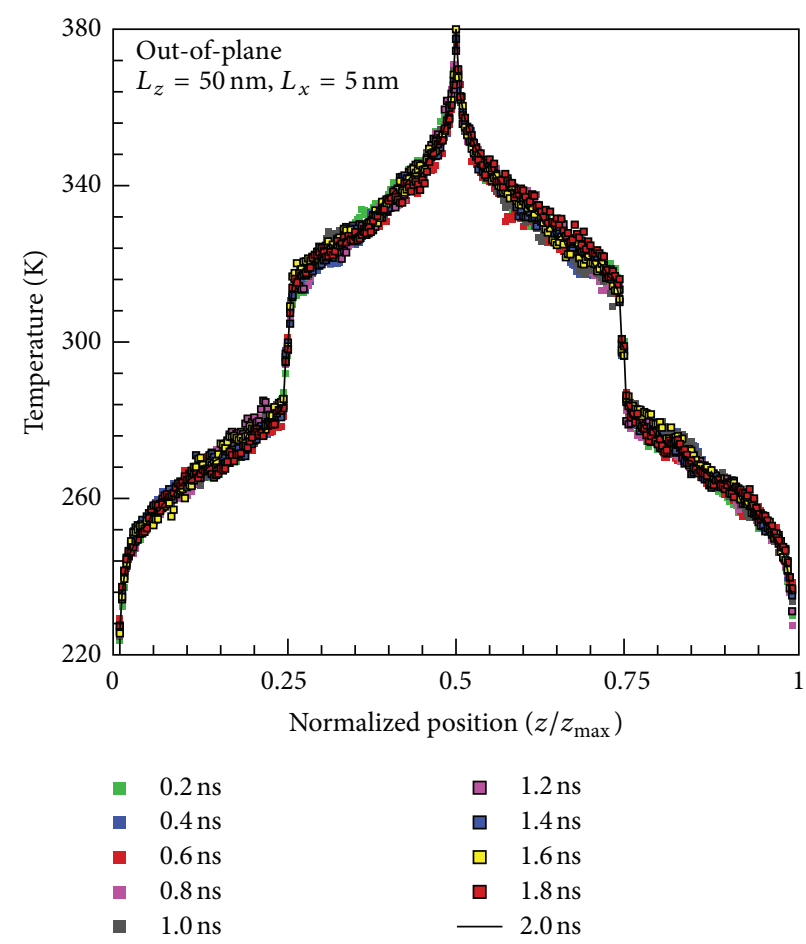

(b)

FIGURE 3: (a) Cumulative energy transported during RNEMD as a function of elapsed simulation time and (b) the evolution of the temperature profile in the simulated structures with time.

Using Fourier's law, the thermal conductivity, $k$, is obtained as

$$
k=-\frac{\langle q\rangle}{\langle d T / d x\rangle},
$$

where $\langle q\rangle$ is the heat flux and $\langle d T / d x\rangle$ is temperature gradient averaged over time and space. From the temperature jumps in the temperature profiles, interfacial thermal resistance, $R$, can be calculated by using

$$
R=\left\langle\frac{\Delta T}{q}\right\rangle .
$$

In the calculation of the thermal conductivity, only areas that directly contribute to thermal transport are used-annular area of one $(6,6)$ SWNCT $(0.335 \mathrm{~nm}$ thickness) is used in the out-of-plane thermal conductivity calculation while sectional areas of two graphene layers (each with $0.335 \mathrm{~nm}$ thickness) are used for the in-plane thermal conductivity calculation, as shown in Figure 4.

\section{Results and Discussion}

Table 1 lists the out-of-plane and in-plane thermal conductivities and the interfacial thermal resistances obtained from all the simulations conducted in the present study. The interpillar distance was varied from $5 \mathrm{~nm}$ to $20 \mathrm{~nm}$ while the pillar height was changed from 25 to $200 \mathrm{~nm}$. To save computational time the in-plane thermal conductivities for unit cells

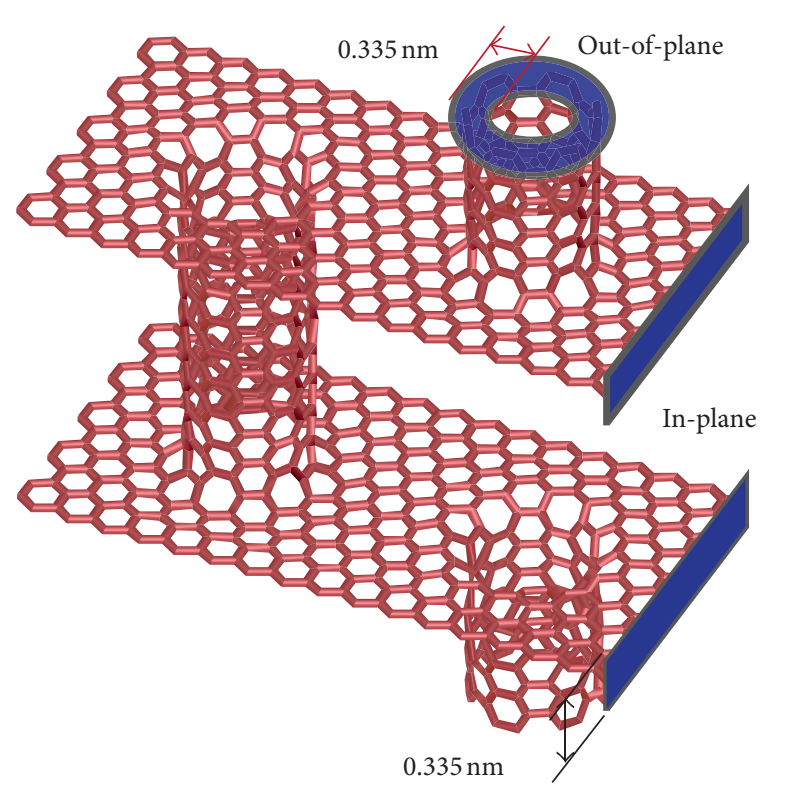

FIGURE 4: Area selection for thermal conductivity calculations in the out-of-plane and in-plane directions.

with $20 \mathrm{~nm}$ interpillar distance and pillar heights of $50 \mathrm{~nm}$ and $100 \mathrm{~nm}$ were omitted. The validation of the simulation methods was provided in our previous work [19] in which the theoretical predictions of the thermal conductivity of pristine $(6,6)$ SWCNT and single layer graphene were compared with experimental measurements reported by other researchers. 


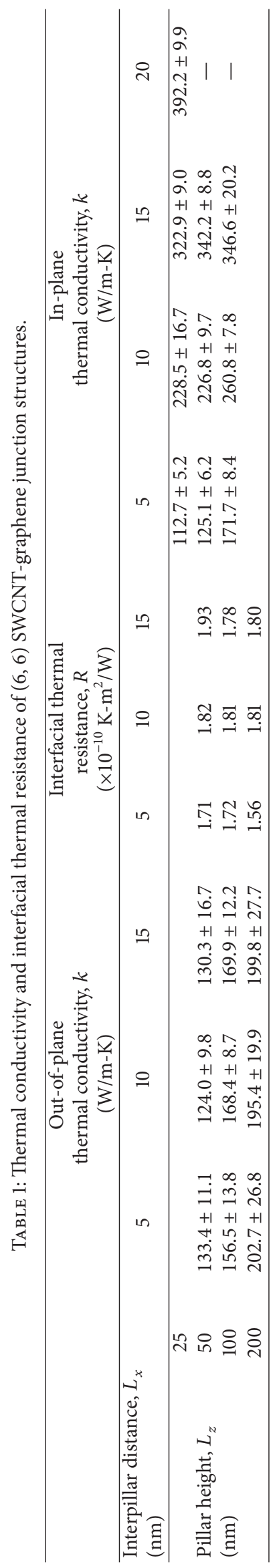




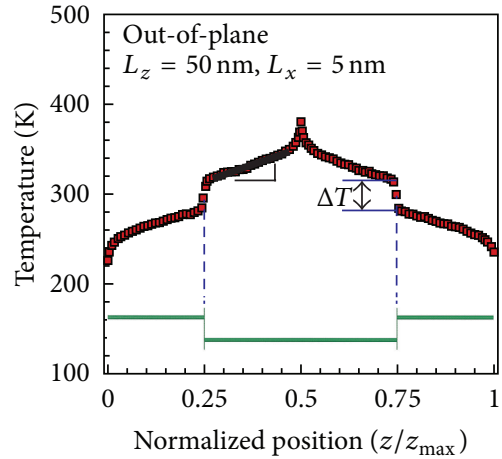

(a)

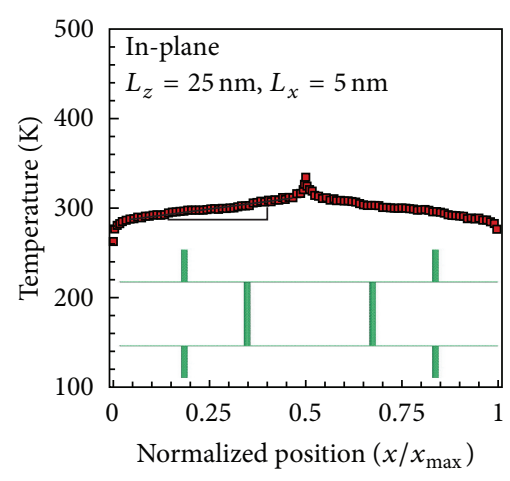

(d)

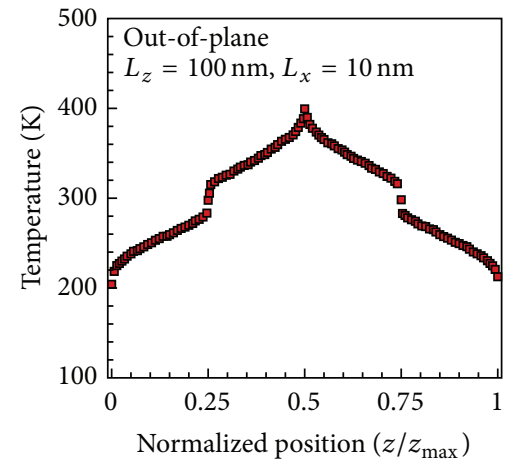

(b)

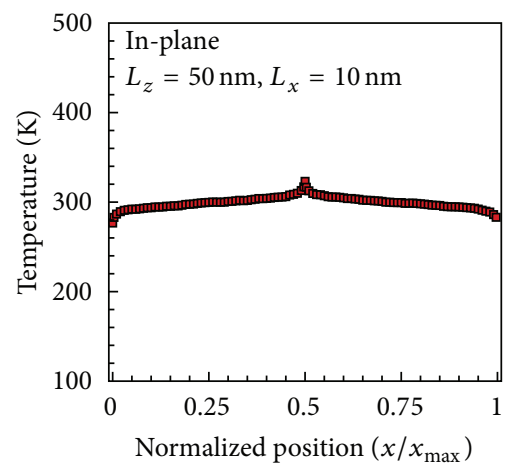

(e)

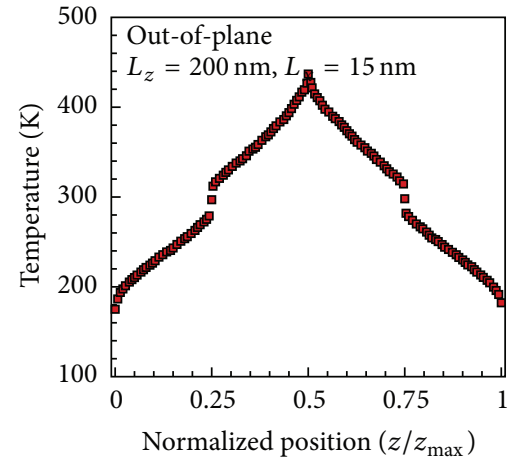

(c)

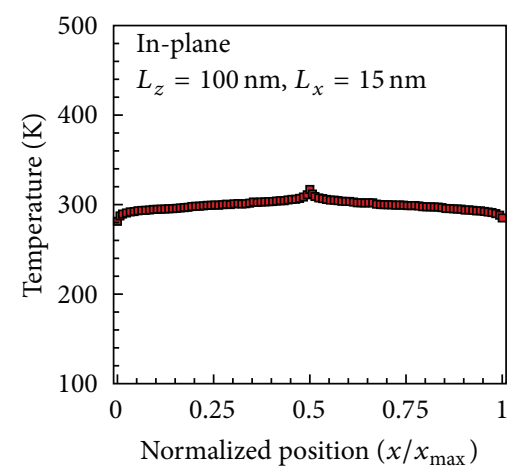

(f)

Figure 5: Temperature profiles obtained from RNEMD simulations in the out-of-plane ((a), (b), and (c)) and in-plane ((d), (e), and (f)) directions. The temperature profiles are used to calculate the thermal interfacial resistance and overall thermal conductivity of the unit cells.

Figure 5(a) shows the temperature profile for out-ofplane thermal conductivity in a unit cell with a $50 \mathrm{~nm}$ pillar height obtained after imposing a heat flux for a total duration of $2 \mathrm{~ns}$. The temperature gradient is determined from the slope of the linear region (shown as a solid black line in Figure 5(a)) excluding the hot and cold bath regions and also excluding the region of the sudden temperature drop. The temperature slips at the specimen boundary are a consequence of quasi-ballistic thermal transport due to the relatively short length of the sample when compared to the phonon mean free path. Fourier theory of thermal transport considers heat transport as a diffusive process where energy flow is driven by a temperature gradient. However, this is not valid at length scales smaller than the mean free path for the energy carriers in a material, which can be tens of nanometers in carbon-based nanomaterials at room temperature. In this case, the heat flow can become ballistic, as seen by the temperature slips near the ends (hot and cold baths) of the simulation box. Distinct temperature jumps can also be observed at the SWCNT-graphene junction locations in the temperature profile. The distinct temperature jumps indicate the existence of an interfacial thermal resistance as heat flows from one SWCNT to the other via the two SWCNT-graphene junctions and the adjoining graphene region.

Based on the observed temperature drops in the temperature profiles, interfacial thermal resistance is calculated for the nine different SWCNT-graphene junction structures and the results are shown in Table 1. The interfacial thermal resistance for out-of-plane thermal transport is observed to decrease slightly with an increase in pillar height. Also, it is observed that the out-of-plane thermal conductivity depends weakly on the interpillar distance. For the case of the in-plane thermal conductivity the temperature gradient is determined from the slope of the linear region (shown as a solid black line in Figure 5(d)) excluding the hot and cold bath regions. It is interesting to note that unlike the case with out-of-plane thermal transport the sudden temperature jumps are not evident at the locations of the SWCNTgraphene junctions in the in-plane temperature profiles. An important implication of this observation is that phonon scattering during in-plane thermal transport is weaker (or nearly absent) when compared to the out-of-plane thermal transport in these SWCNT-graphene hybrid structures. From the linear regression of the slope, equivalent in-plane thermal conductivities for 10 different SWCNT-graphene junction structures are calculated, and the results are shown in Table 1. The in-plane thermal conductivity shows a small dependency on pillar height and the effect becomes smaller as the interpillar distance increases.

The slight decrease in the interfacial thermal resistance with an increase in SWCNT pillar height during out-ofplane thermal transport can be attributed to scattering of low frequency (i.e., large wavelength) phonons [23, 24]. On the other hand, the reasons for the slight increase in inplane thermal conductivity with an increase in SWCNT pillar height when the interpillar distance is small $(5 \mathrm{~nm})$ are much 


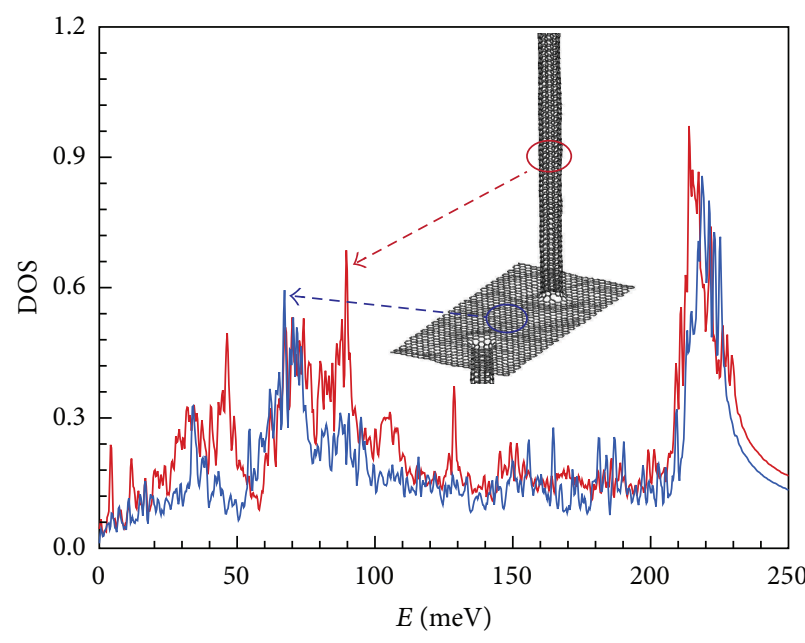

(a)

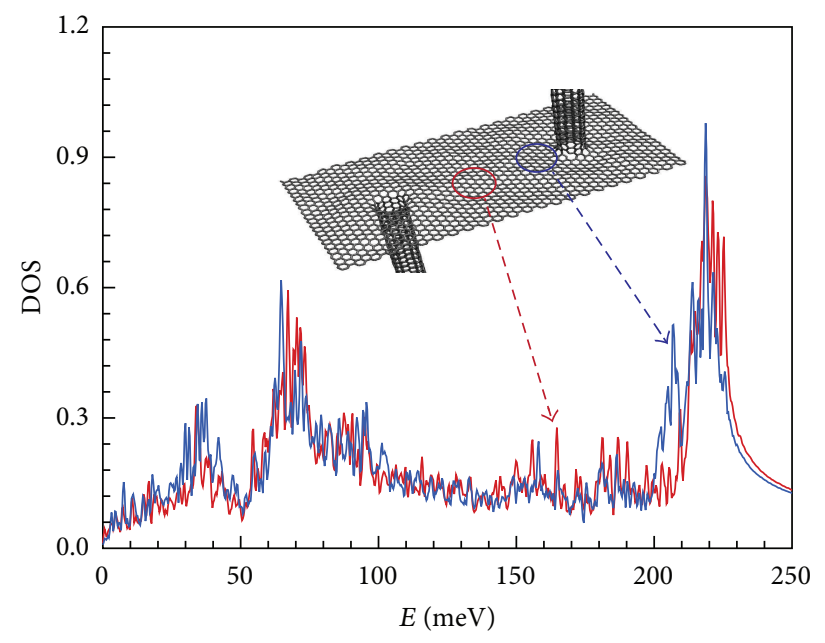

(b)

FIGURE 6: Local density of states (LDOS) calculated by velocity autocorrelation of atoms in the selected region of the unit cells. (a) Comparison of the LDOS obtained from a region of the SWCNT away from the junction and in graphene floor away from the junction. (b) Comparison of the LDOS obtained from a region of the graphene floor close to the junction and away from the junction.

more complex. Further studies, including the wave packet analysis, will be undertaken in the future to better understand these effects. However, the small dependency of in-plane thermal conductivity on pillar height when the interpillar distance is large can be attributed to the near diffusive thermal transport in graphene in the region between the junctions. Along the same vein, Bagri et al. [20] studied the effect of twin grain boundary on the thermal transport of graphene and showed a similar trend in thermal conductivity with different graphene lengths in between the defected regions. They showed that the thermal conductivities of graphene with 13.2-degree grain boundary orientation were higher than the ones with 5.5-degree grain boundary orientation for structures with relatively short graphene lengths between defects ( $25 \mathrm{~nm}$ and $50 \mathrm{~nm}$ ) but could not observe a clear difference between the thermal conductivities for structures with 13.2degree grain boundary orientation and the 5.5-degree grain boundary orientation when the distance between defects was relatively larger $(125 \mathrm{~nm})$.

With regard to the distinct temperature jumps in the temperature profiles (Figure 5) during out-of-plane thermal transport, we hypothesize that the jumps correspond to phonon dispersion due to the sudden change in dimensionality which the phonons experience as they flow from the quasi-1D structure (SWCNT) to 2D (graphene) and then back again to quasi-1D (SWCNT) via the SWCNTgraphene junctions. As a consequence, the low frequency longitudinal acoustic phonon modes, which carry thermal energy efficiently in a SWCNT, must transform into phonon modes which are efficient energy carriers of thermal energy in graphene, for example, the in-plane modes, and then back to the preferred phonon modes in SWCNTs. In order to better understand the contributions of SWCNT-graphene junctions we investigate the local phonon density of states (LDOS) using velocity autocorrelation along the phonon transport path. It is expected that the subtle differences in the LDOS in various regions along the path of the phonon transport, that is, the SWCNT-graphene junctions, graphene floor, and the adjoining SWCNTs, would help to provide insights into the origin of thermal interfacial resistance at the SWCNTgraphene interface in these systems. For example, a mismatch in the LDOS in the SWCNT and the graphene regions would represent a mismatch in the phonon spectra between the two regions and hence to a higher thermal resistance across the interface.

Figure 6(a) shows the LDOS in the SWCNT and the graphene floor from regions away from the SWCNTgraphene junction. It can be observed that the LDOS distribution is similar except in the low energy (frequency) regime between $60 \mathrm{meV}$ and $110 \mathrm{meV}$. Since these low energy (frequency) phonon modes are expected to contribute more to the thermal transport characteristics when compared to the high energy (frequency) phonon modes, the change in dimensionality from $1 \mathrm{D}$ to $2 \mathrm{D}$ to $\mathrm{ID}$ is expected to contribute to the observed thermal interfacial resistance during out-ofplane thermal transport in these structures. On the other hand, the LDOS for in-plane thermal transport in regions close to and away from the SWCNT-graphene junctions is shown in Figure 6(b). It can be observed that the LDOS in the two regions is very similar except for the higher energy (frequency) phonon modes with energy greater than $190 \mathrm{meV}$. Since most of the higher energy phonons reside in the higher frequency optical modes which have relatively low group velocities they are expected to contribute little to the overall thermal conductivity and hence to a negligible interfacial thermal resistance.

Figure 7 shows a comparison of the thermal conductivity of the 3D SWCNT-graphene hybrid structure with the thermal conductivity of pristine $(6,6)$ SWCNT and single layer graphene of corresponding size. In Figure 7(a) thermal conductivities of individual $(6,6)$ SWCNT are compared with out-of-plane thermal conductivities of SWCNT-graphene 


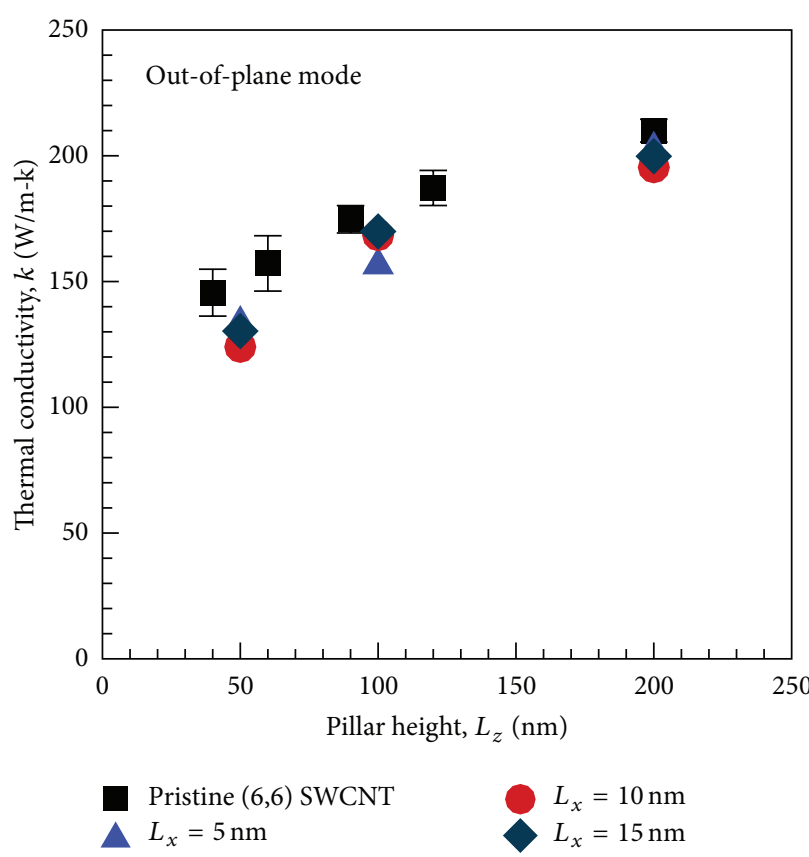

(a)

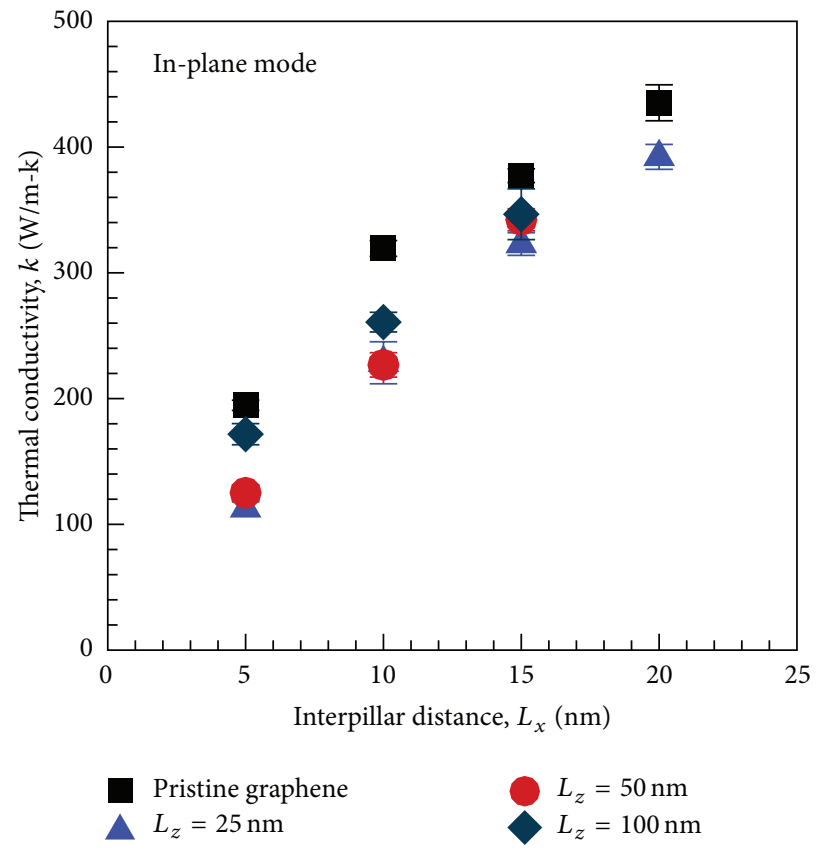

(b)

FIGURE 7: (a) Comparison of the thermal conductivity of $(6,6)$ SWCNT and out-of-plane thermal conductivity of SWCNT-graphene hybrid structures as a function of pillar height. (b) Comparison of the thermal conductivity of single layer graphene and in-plane thermal conductivity of the 3D SWCNT-graphene hybrid structures as a function of the interpillar distance.

hybrid structures. The out-of-plane thermal conductivity of the 3D hybrid structures is observed to increase with increasing size of the unit cell, that is, with an increase in pillar height (length of the SWCNT). It is interesting to note that the increase in out-of-plane thermal conductivity in SWCNT-graphene hybrid structure is similar to that observed in pristine $(6,6)$ SWCNT. In our previous study of thermal transport in (6.6) SWCNTs [19] we observed that thermal conductivity of SWCNTs is linearly dependent on specimen length as long as the length of the SWCNTs is smaller than the mean free path (ballistic thermal transport) and then transitions to a regime where it becomes nearly length-independent (diffusive and governed by Fourier's law). Consequently, the out-of-plane thermal conductivity of the 3D hybrid structures is expected to occur nearly linearly with pillar height as long as the height is smaller than the critical length for ballistic thermal transport in $(6,6)$ SWCNT and then approaches a plateau as the pillar height becomes greater than the ballistic length $\sim 75 \mathrm{~nm}$. In addition, due to the presence of interfacial thermal resistance at the SWCNTgraphene junction, the thermal conductivity of the hybrid structure is consistently lower than that of pristine $(6,6)$ SWCNT. As seen in the temperature profiles describing the out-of-plane thermal transport (Figures 5(a), 5(b), and 5(c)), thermal vibrations must travel from SWCNT via SWCNTgraphene junctions to reach the next SWCNT; this leads to an approximately $5 \%$ decrease in thermal conductivity in the out-of-plane direction.

Figure 7(b) compares thermal conductivity of single layer graphene with in-plane thermal conductivity obtained from simulations using various sized SWCNT-graphene hybrid structures. Like in the case of out-of-plane thermal conductivity, the thermal conductivities of the hybrid structures are observed to increase linearly with an increase in cell size (interpillar distance). It is postulated that the increase in thermal conductivity with interpillar distance is primarily due to the size of the structures evaluated in the present paper, which are smaller than the critical ballistic length in single layer graphene for a given graphene floor width. Moreover, since the hybrid structures investigated herein are created such that the width of graphene layer $\left(L_{y}\right)$ is the same as the interpillar distance $\left(L_{x}\right)$, it is expected that the ratio of defected (junction) area to total graphene area becomes smaller as the interpillar distance becomes larger. Use of larger sized structures (greater than the critical ballistic length in graphene) in the present analysis becomes computationally prohibitive. As the interpillar distance increases, it is expected that the difference between the in-plane thermal conductivities of the $3 \mathrm{D}$ hybrids and the single layer graphene (of corresponding size) converges.

In summary, in the present paper we present results of a reverse nonequilibrium molecular dynamics study with AIREBO interatomic potential to investigate thermal transport in 3D SWCNT-graphene hybrid structures comprising SWCNT-graphene junctions. The results of the simulations reveal the presence of interfacial thermal resistance for thermal transport in the out-of-plane direction (along the axis of the SWCNT pillars) while the thermal resistance along the graphene floor (in-plane direction) is found to be negligible. The origin of thermal resistance in the outof-plane direction is understood to be a combination of phonon scattering due to the change in dimensionality of 
the medium along the phonon path (i.e., from quasi-1D in SWCNT to $2 \mathrm{D}$ in graphene) and a mismatch of phonon spectra along the phonon transport path. In addition, the interfacial thermal resistance is found to be weakly dependent on the pillar (SWCNT) height as well as the interpillar distance. For out-of-plane thermal transport the highest interfacial thermal resistance across the SWCNT-graphene junction was computed to be $1.93 \times 10^{-10} \mathrm{~K}-\mathrm{m}^{2} / \mathrm{W}$ for a pillar height of $50 \mathrm{~nm}$ and an interpillar distance of $15 \mathrm{~nm}$. Moreover, the highest out-of-plane thermal conductivity of a unit cell representing the SWCNT-graphene hybrid structure was obtained to be $202.7 \pm 26.8 \mathrm{~W} / \mathrm{m}-\mathrm{K}$ for a cell with pillar height of $200 \mathrm{~nm}$ and an interpillar distance of $5 \mathrm{~nm}$. In comparison the thermal conductivity of a pristine $(6,6)$ SWCNT with $200 \mathrm{~nm}$ length is computed to be $209.9 \pm$ $4.5 \mathrm{~W} / \mathrm{m}-\mathrm{K}$. The highest in-plane thermal conductivity of a unit cell with two SWCNT-graphene junctions and a cell size of $60 \mathrm{~nm}$ with $25 \mathrm{~nm}$ pillar height was estimated to be $392.2 \pm 9.9 \mathrm{~W} / \mathrm{m}-\mathrm{K}$; the thermal conductivity of pristine single layer graphene with the same dimensions was computed to be $435.2 \pm 14.3 \mathrm{~W} / \mathrm{m}-\mathrm{K}$. Moreover, the thermal conductivity of the hybrid unit cells was observed to increase nearly linearly with an increase in dimensions of the cell, that is, pillar height as well as interpillar distance. The thermal conductivity of the cell is observed to approach a plateau as the pillar height and the interpillar distance approach the critical lengths for ballistic thermal transport in SWCNT and single layer graphene of comparable sizes. These results indicate that the out-of-plane and in-plane thermal transport characteristics of these 3D SWCNT-graphene hybrid structures can be tuned by controlling the unit cell size.

\section{Conflict of Interests}

The authors declare that there is no conflict of interests regarding the publication of this paper.

\section{Acknowledgments}

This work made use of the High Performance Computing Resource in the Core Facility for Advanced Research Computing at Case Western Reserve University. The authors also would like to acknowledge the support of the Air Force Office of Scientific Research (AFOSR) MURI Grant no. FA955012-1-0037 (Program Manager: Dr. Joycelyn Harrison) for conducting this research.

\section{References}

[1] V. Varshney, S. S. Patnaik, A. K. Roy, G. Froudakis, and B. L. Farmer, "Modeling of thermal transport in pillared-graphene architectures," ACS Nano, vol. 4, no. 2, pp. 1153-1161, 2010.

[2] L. Xu, N. Wei, Y. Zheng, Z. Fan, H. Wang, and J. Zheng, "Graphene-nanotube 3D networks: intriguing thermal and mechanical properties," Journal of Materials Chemistry, vol. 22, no. 4, pp. 1435-1444, 2012.

[3] Z. Fan, J. Yan, L. Zhi et al., "A three-dimensional carbon nanotube/graphene sandwich and its application as electrode in supercapacitors," Advanced Materials, vol. 22, no. 33, pp. 3723$3728,2010$.

[4] G. K. Dimitrakakis, E. Tylianakis, and G. E. Froudakis, "Pillared graphene: a new 3-D network nanostructure for enhanced hydrogen storage," Nano Letters, vol. 8, no. 10, pp. 3166-3170, 2008.

[5] E. Tylianakis, G. M. Psofogiannakis, and G. E. Froudakis, "Lidoped pillared graphene oxide: a graphene-based nanostructured material for hydrogen storage," The Journal of Physical Chemistry Letters, vol. 1, no. 16, pp. 2459-2464, 2010.

[6] F. D. Novaes, R. Rurali, and P. Ordejón, "Electronic transport between graphene layers covalently connected by carbon nanotubes," ACS Nano, vol. 4, no. 12, pp. 7596-7602, 2010.

[7] S. Li, Y. Luo, W. Lv et al., "Vertically aligned carbon nanotubes grown on graphene paper as electrodes in lithium-ion batteries and dye-sensitized solar cells," Advanced Energy Materials, vol. 1, no. 4, pp. 486-490, 2011.

[8] J. Lee, V. Varshney, A. K. Roy, and B. L. Farmer, "Single mode phonon energy transmission in functionalized carbon nanotubes," Journal of Chemical Physics, vol. 135, no. 10, Article ID 104109, 2011.

[9] Y. Zhu, L. Li, C. Zhang et al., "A seamless three-dimensional carbon nanotube graphene hybrid material," Nature Communications, vol. 3, article 1225, 2012.

[10] G. C. Loh, E. H. T. Teo, and B. K. Tay, "Interpillar phononics in pillared-graphene hybrid nanostructures," Journal of Applied Physics, vol. 110, no. 8, Article ID 083502, 2011.

[11] W. Humphrey, A. Dalke, and K. Schulten, "VMD: visual molecular dynamics," Journal of Molecular Graphics, vol. 14, no. 1, pp. 33-38, 1996.

[12] J. González, F. Guinea, and J. Herrero, "Propagating, evanescent, and localized states in carbon nanotube-graphene junctions," Physical Review B-Condensed Matter and Materials Physics, vol. 79, no. 16, Article ID 165434, 2009.

[13] J. González and J. Herrero, "Graphene wormholes: a condensed matter illustration of Dirac fermions in curved space," Nuclear Physics B, vol. 825, no. 3, pp. 426-443, 2010.

[14] D. L. Nika and A. A. Balandin, "Phonon transport in graphene," http://arxiv.org/abs/1203.4282.

[15] J.-C. Charlier, T. W. Ebbesen, and P. Lambin, "Structural and electronic properties of pentagon-heptagon pair defects in carbon nanotubes," Physical Review B-Condensed Matter and Materials Physics, vol. 53, no. 16, pp. 11108-11113, 1996.

[16] J. Park, M. F. P. Bifano, and V. Prakash, "Sensitivity of thermal conductivity of carbon nanotubes to defect concentrations and heat-treatment," Journal of Applied Physics, vol. 113, no. 3, Article ID 034312, 2013.

[17] J. Ma, D. Alfè, A. Michaelides, and E. Wang, "Stone-Wales defects in graphene and other planar $s p^{2}$-bonded materials," Physical Review B-Condensed Matter and Materials Physics, vol. 80, no. 3, Article ID 033407, 2009.

[18] F. Müller-Plathe, "A simple nonequilibrium molecular dynamics method for calculating the thermal conductivity," Journal of Chemical Physics, vol. 106, no. 14, pp. 6082-6085, 1997.

[19] J. Park and V. Prakash, "Thermal transport in 3D pillared SWCNT-graphene nanostructures," Journal of Materials Research, vol. 28, no. 7, pp. 940-951, 2013.

[20] A. Bagri, S. Kim, R. S. Ruoff, and V. B. Shenoy, "Thermal transport across twin grain boundaries in polycrystalline graphene from nonequilibrium molecular dynamics simulations," Nano Letters, vol. 11, no. 9, pp. 3917-3921, 2011. 
[21] S. Plimpton, P. Crozier, and A. Thompson, LAMMPS-LargeScale Atomic/Molecular Massively Parallel Simulator, Sandia National Laboratories, 2007.

[22] S. J. Stuart, A. B. Tutein, and J. A. Harrison, "A reactive potential for hydrocarbons with intermolecular interactions," Journal of Chemical Physics, vol. 112, no. 14, pp. 6472-6486, 2000.

[23] H. Zhong and J. R. Lukes, "Interfacial thermal resistance between carbon nanotubes: molecular dynamics simulations and analytical thermal modeling," Physical Review BCondensed Matter and Materials Physics, vol. 74, no. 12, Article ID 125403, 2006.

[24] S. T. Huxtable, D. G. Cahill, S. Shenogin et al., "Interfacial heat flow in carbon nanotube suspensions," Nature Materials, vol. 2, no. 11, pp. 731-734, 2003. 

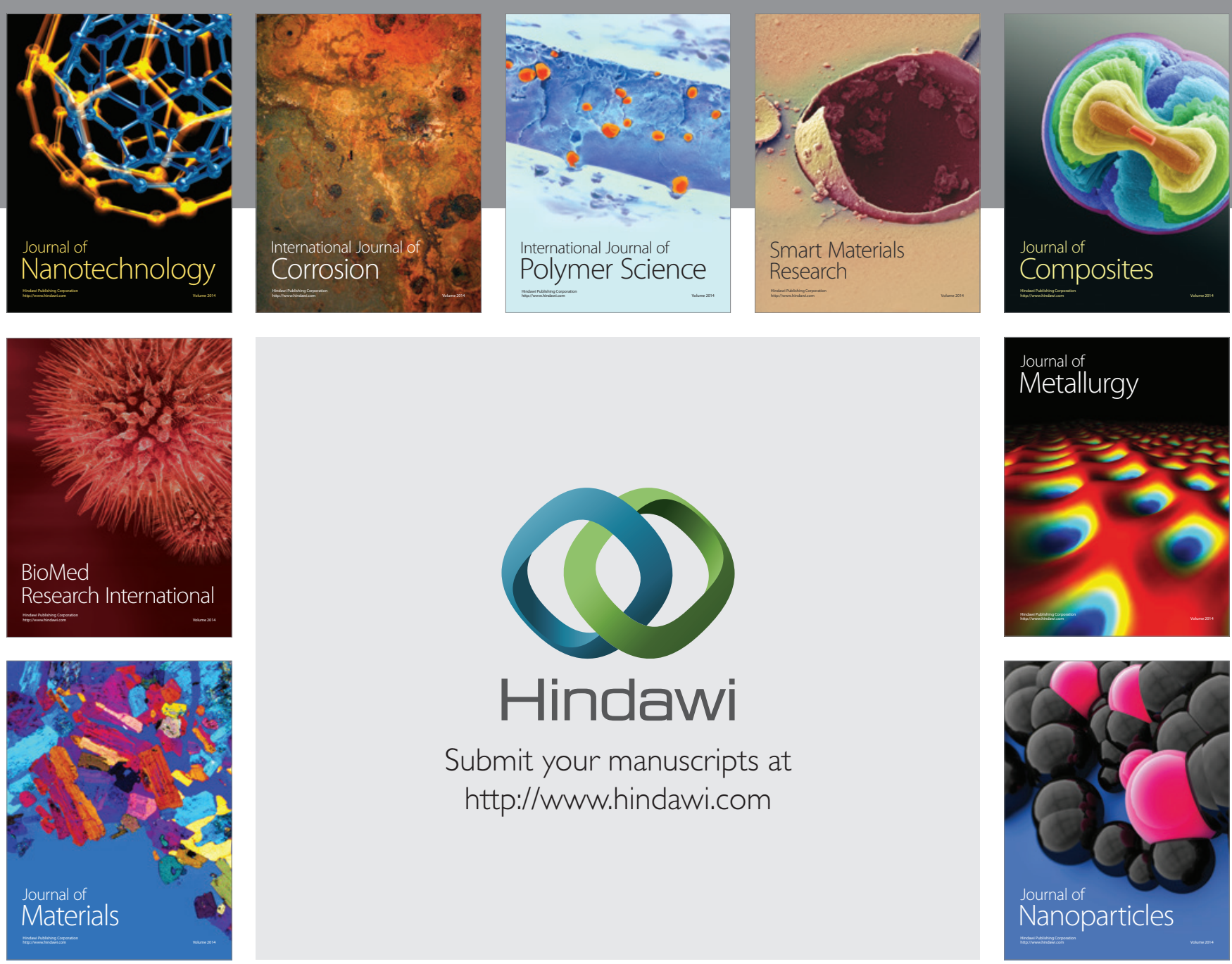

Submit your manuscripts at http://www.hindawi.com
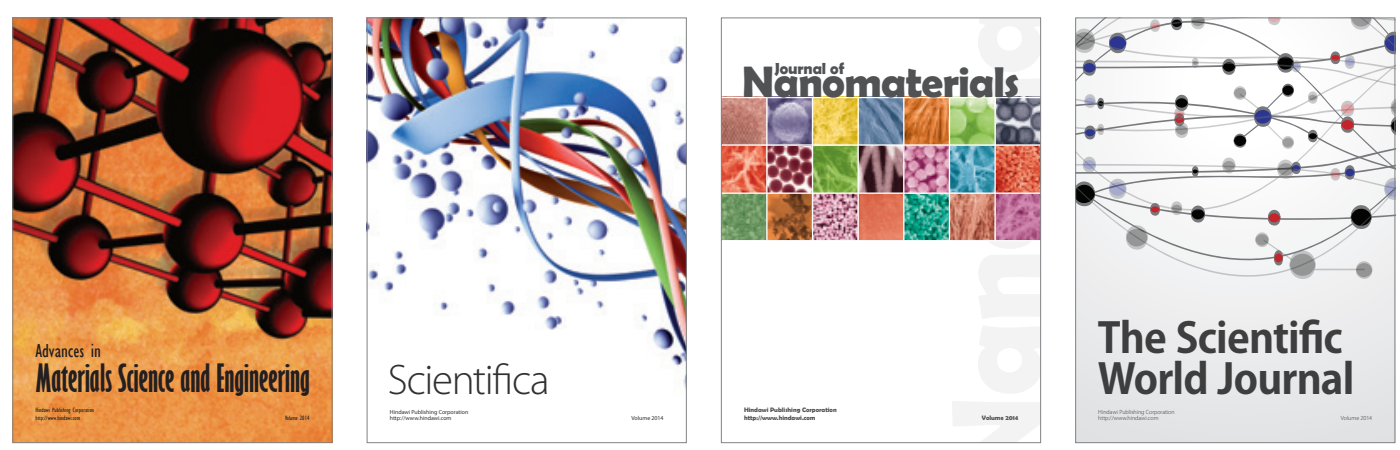

\section{The Scientific World Journal}
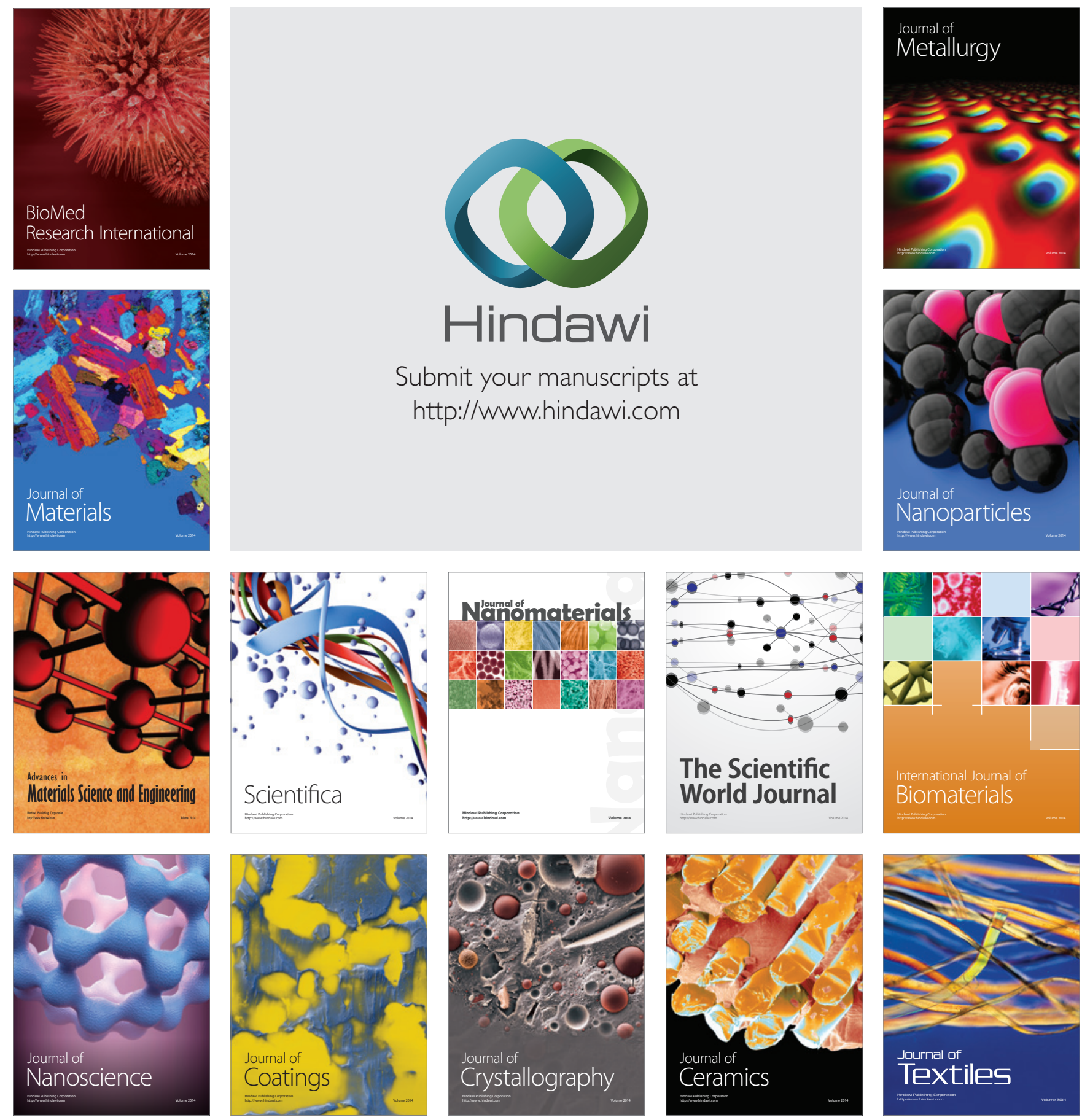